\title{
REVISION DE LOS GENEROS HYPERPHYSCIA, PHAEOPHYSCIA, PHYSCIA Y PHYSCONIA EN EL SUR DE ESPAÑA
}

\author{
J.G. PEDREÑO, P.P. MORENO \& J.M. EGEA
}

RESUMEN: Se citan 21 especies y una subespecie de los géneros Hyperphyscia, Phaeophyscia, Physcia y Physconia reconocidas hasta la fecha en el S.E. de España. Se incluye una clave dicotómica con las especies consideradas, asi como ilustraciones que recogen los principales caracteres utilizados en la delimitación de los distintos táxones.

ABSTRACT: 21 taxa of genera Hyperphyscia, Phaeophyscia, Physcia and Physconia from South East Spain are cited. A dichotomous key with those species, and illustrations on main characters used to delimit taxa are furnished.

\section{INTRODUCCION}

El género Physcia s., desde la fecha de su publicación (Michaux, 1803), ha sido objeto de varias revisiones taxonómicas, centradas en el Norte y Centro de Europa: Noruega (Lynge, 1916); Alemania, Austria, Suiza (Lynge, 1935); Checoslovaquia (Nadvornik, 1947); Holanda (Maas Geesteranus, 1952 ); Suiza (Frey, 1963). Thomson (1963) publica una monografía del género en América del Norte.

En los estudios anteriores se admite un solo género (Physcia), subdividido en varios táxones infragenéricos. Los criterios utilizados para la delimitación de los grupos se basan en: aspecto general del talo, color de la cara superior e inferior, presencia o ausencia de pruinas, estructuras vegetativas (fibrillas, diásporas), y reacción del córtex superior frente a la potasa (K).

Poelt (1965) hace una nueva revisión de la familia Physciaceae, e introduce como caracteres diferenciales de gran importancia taxonómica: el tipo de ascósporas, estructura del córtex, y tipo de rizinas. En base a estos caracteres, propone un nuevo género: Physconia, y admite el género Physciopsis, creado por Choisy (1950) y no aceptado en trabajos anteriores. Años más tarde, este mismo autor (Poelt, 1974) realiza un estudio de los géneros anteriores en Himalaya, y Kashiwadani (1975) publica los resultados obtenidos en Japón.

Moberg (1977), en su monografía del género Physcia y otros géneros relacionados en Escandinavia, añade a los criterios taxonómicos utilizados por Poelt el tipo de picnoconidios y la 
composición quimica. Admite los géneros propuestos por Poelt y describe el nuevo género Phaeophyscia.

Mayrhoffer \& Poelt (in Hafellner et al., 1979) incluyen el género Phaeophyscia dentro de Physcia, y sinonimizan Physciopsis a Hyperphyscia, taxon propuesto por Müller a finales del siglo pasado (Müller, 1884), y no tenido en cuenta hasta la fecha.

Todos los trabajos mencionados se han realizado fuera de la región Mediterránea. 'E1 estudio que presentamos es una revisión de los géneros Hyperphyscia, Phaeophyscia, Physcia y Physconia en el S.E. de España. Los resultados obtenidos pueden extrapolarse, en gran parte, al resto de la Peninsula Ibérica y Norte de Africa.

\section{MATERIAL Y METODOS}

El material examinado procede de diversas campañas de prospecciones liquenológicas realizadas desde 1975 por algunos miembros del Departamento de Botánica en las provincias de Murcia, Albacete, Almeria, Jaén y Granada, y conservadas en el Herbario de Liquenes de la Universidad de Murcia (MUB-Liquenoteca).

Para la correcta determinación de los ejemplares se ha utilizado la bibliografía clásica: Lynge (1935), Poelt (1969), Ozenda \& Clauzade (1970). Además, se han empleado monografías y separatas sobre aspectos parciales del tema del presente trabajo: Poelt (1974), Kashiwadani (1975), Moberg (1974, 1975, 1977 ), etc.

\section{DELIMITACION DE GENEROS}

Para delimitar los distintos grupos, se han utilizado fundamentalmente las siguientes caracteristicas taxonómicas:

- Tipo de rizinas: simples y escuarrosas (Fig. 1).

- Tipo de ascósporas: Physcia, Physconia y Pachysporaria (Fig. 2 A-D).

- Presencia y estructura de isidios, pseudoisidios y soralios (fig. 2 E-F).

- Tipo de picnidios: Umbilicaria, Xanthoria y Roccella (Fig. 3).

- Tipo de picnoconidios: cilíndrico, elipsoidal y filiforme (Fig. 3)

- Sustancias liquénicas: atranorina, skirina y zeorina.

- Tipo de talo: de fuerte a laxamente adherido al sustrato.

En la Tabla I aparecen las caracteristicas de los géneros considerados en el presente trabajo.

\section{CLAVE DE ESPECIES}

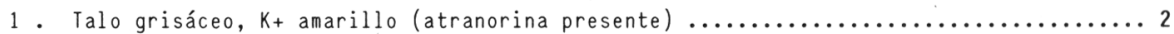

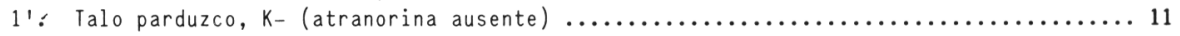

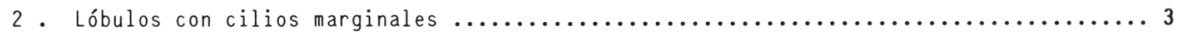

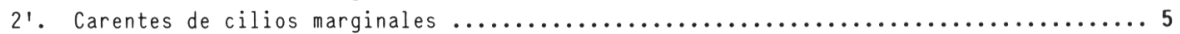

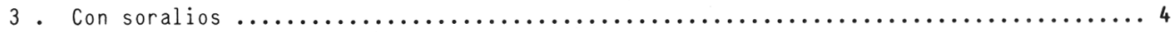

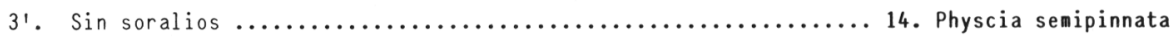


4. Con soralios en capuchón

5. Médula $K_{+}($amarillo) 6

5'. Médula K- 8

6 . Soralios labriformes

10. Physcia caesia

6'. Sin soralios 7

7. Con pseudoisidios

11. Physcia clementei

7'. Sin pseudoisidios, y punteada de blanco 8. Physcia aipolia

8. Con soralios 12. Physcia dubia

$8^{\prime}$. Sin soralios 10

9. Talo densamente pruinoso

9'. Talo sin o con poca pruina

15. Physcia stellaris

10 . Esporas ornamentadas

13. Physcia magnussonii

10'. Esporas no ornamentadas 9. Physcia biziana

11. Talo muy adherido al sustrato, con pocas o sin rizinas; picnonidios filiformes

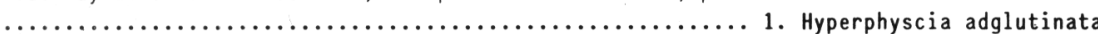

11'. Talo con abundantes rizinas; picnonidios subcilíndricos o elipsoidales ............ 12

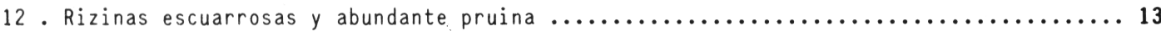

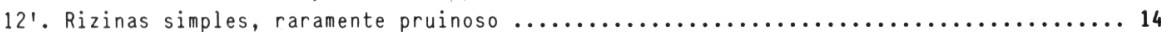

13. Médula $K_{+}\left(\right.$amarillo), soralios $K_{+} \ldots \ldots \ldots \ldots \ldots \ldots \ldots \ldots \ldots \ldots \ldots \ldots \ldots$ Physconia enteroxantha

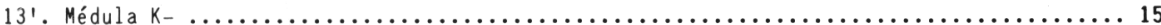

14 . Con pruina blanca, cara inferior generalmente blancuzca ................... 14'. Sin pruina o muy escasa y parda; cara inferior de parda a negra, raramente blancuzca ...

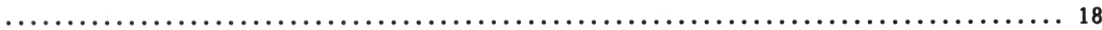

15 . Con soralios labriformes

19. Physconia perisidiosa

15'. Sin soralios

16 . Apotecios con reborde talino provisto de proliferaciones foliáceas muy abundantes .....

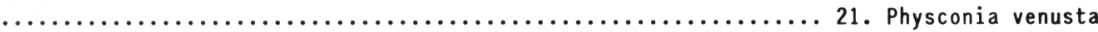

16'. Apotecios con proliferaciones foliáceas poco desarrolladas, o sin ellas ............. $\ldots \ldots \ldots \ldots \ldots \ldots \ldots \ldots \ldots \ldots \ldots \ldots \ldots \ldots \ldots \ldots \ldots \ldots \ldots$. Physconia pulverulacea

17. Lóbulos de más de $2 \mathrm{~mm}$ de anchura ............... 18a. Physconia grisea subsp. grisea

17'. Lóbulos de menos de $2 \mathrm{~mm}$ de anchura; de tonalidad verde-violácea cuando se humedece .... $\ldots \ldots \ldots \ldots \ldots \ldots \ldots \ldots \ldots \ldots \ldots \ldots \ldots \ldots \ldots \ldots \ldots \ldots \ldots . \ldots \ldots$. Physconia grisea subsp. lilacina

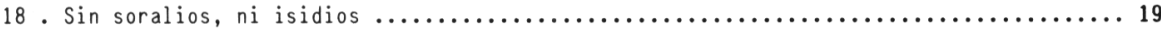

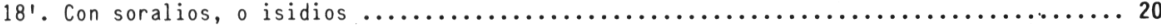

19. Médula blanca, K-; apotecios con abundantes rizinas en corona; cara inferior blanca con

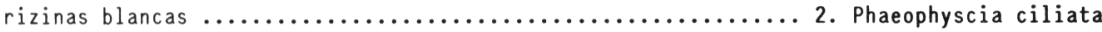

19'. Médula $K_{+}($rojo) o blanca; apotecios sin corona de rizinas; cara inferior negra, con

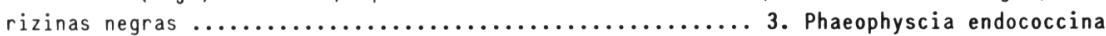

20 . Soralios maculiformes 


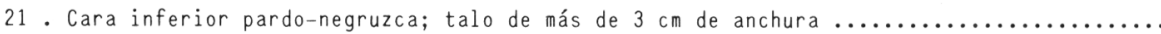

211. Cara inferior blanquecina;

4. Phaeophyscia insignis

\section{CATALOGO}

1. Hyperphyscia adglutinata (Flk.) Mayrh. \& Poelt

MURCIA: Sa de Carrascoy, Rambla de Roy, 700-900 m (MUB 6026); Bullas, Carrascalejo, 600 m (MUB 6025); Jumilla. Sa del Carche, Barranco del Revolcador, $900 \mathrm{~m}$ (MUB 6024).

En la Península Ibérica se ha encontrado exclusivamente en cortezas de planifolios; sin embargo, este taxon puede comportarse también como saxicola. Nitrófilo. Sobre Quercus rotundifolia.

El córtex inferior es muy difícil de distinguir, ya que el talo aparece fuertemente adherido al sustrato. En algunos se encuentran ascósporas de longitud ligeramente inferior a la considerada por Moberg (1977).

\section{Phaeophyscia ciliata (Hoff.) Moberg}

ALBACETE: Yeste, Casa del Barbero, $700 \mathrm{~m}$ (MUB 7654).

Corticicola, óptimo desarrollo sobre cortezas más o menos ácidas y ricas en nutrientes; raramente se ha encontrado en otro tipo de sustrato. Sobre Populus nigra.

3. Phaeophyscia endococcina (Kbr.) Moberg

ALMERIA: Gérgal, Sa de los Filabres, Arroyo Verruga, $1900 \mathrm{~m}$ (MUB 1578).

Saxícola, ocasionalmente se ha encontrado sobre cortezas (Moberg, 1977). Generalmente se comporta como acidófilo, hidrófilo, heliófilo, de más o menos fotófilo a esciófilo, en lugares poco o nada nitrificados. El único registro que se tiene de este taxon en España corresponde a rocas ácidas, en paredes más o menos verticales, orientadas al norte, en zonas donde se acumula la humedad atmosférica.

4. Phaeophyscia insignis (Mereschk.) Moberg

ALBACETE: Riópar, Casa de la Noguera, $1000 \mathrm{~m}$ (MUB 7457).

ALMERIA: Mojácar, La Adelfa, 500 m (MUB 1579).

Sobre cortezas eutrofizadas, conviviendo muchas veces con

Ph. orbicularis, siendo Ph. insignis la que puede localizarse en cortezas más oligotróficas, y más o menos ácidas (Crespo \& Bueno, 1982). Nosotros la hemos recogido sobre Quercus rotundifolia y, en Mojácar, sobre micaesquistos.

Taxon muy relacionado con Ph. orbicularis. En base al estudio realizado sobre ejemplares recolectados en el S.E., pueden destacarse las siguientes diferencias:

Ph. insignis

Talo menor de $2 \mathrm{~cm}$

Lóbulos cortos y estrechos, bastante

divididos y adheridos al sustrato

Cara inferior blanquecina

Rizinas blancas y escasas

Apotecios escasos o inexistentes

Picnidios escasos o inexistentes
Ph. orbicularis

Talo mayor de $3 \mathrm{~cm}$

Lóbulos más anchos, imbricados y planos,

algo ascendentes en el ápice

Cara inferior de pardo oscura a negra

Rizinas negras y abundantes

Apotecios abundantes

Picnidos abundantes 
5. Phaeophyscia orbicularis (Neck.) Moberg

ALBACETE: Riópar, Carretera de los Chorros, 1000 m (MUB 7456); Riópar, Arroyo de la Puerta, 1200 m (MUB 7454); Riópar, Casa de la Noguera, $1000 \mathrm{~m}$ (MUB 7455); Yeste, Casa del Barbero, $700 \mathrm{~m}$ (MUB 7611). ALMERIA: Gérgal, Sa de los Filabres, Arroyo Verruga, $1900 \mathrm{~m}$ (MUB 771). JAEN: Sa de Segura, Campamento Rio Madera, $1300 \mathrm{~m}$ (MUB 7585); Sa de Segura, Collado Asperones, $1200 \mathrm{~m}$ (MUB 7538); Sạ de Segura, Molino del Prado de la Porra, $1200 \mathrm{~m}$ (MUB 7528, 7645). MURCIA: Sa de Carrascoy, Rambla de Roy, $900 \mathrm{~m}$ (MUB 6018,6020); Bullas, Salto Lucero, 600 m (MUB 6019 ); Cehegín, Venta del Pino, $800 \mathrm{~m}$ (MUB 6015); Bullas, El Carrascalejo, $600 \mathrm{~m}$ (MUB 6016, 6017).

Indiferente al sustrato, aunque se comporta en gran medida como corticícola; nitrófilo; fotófilo. Sobre Pinus nigra, Quercus rotundifolia, Q. faginea, Populus nigra y Juglans regia.

6. Phaeophyscia sciastra (Ach.) Moberg

ALMERIA: Gérgal, Sạ de los Filabres, Piedra del Sombrero, 2000 m (MUB 1576).

Saxicola, frecuente sobre rocas ácidas; también aparece comúnmente sobre troncos.

7. Physcia adscendens (Fr.) H. Olivier

ALBACETE: Riópar, Cañada de los Mojones, 1300 m (MUB 7442); Riópar, Umbría Fuente Raigadas, $1400 \mathrm{~m}$ (MUB 7441); Riópar, Casa de la Noguera, 1000 m (MUB 7439); Riópar, Chorros del Mundo, $1000 \mathrm{~m}$ (MUB 7616). AlMERIA: Adra, La Alcazaba, $300 \mathrm{~m}$ (MUB 754); Gérgal, Sa de los Filabres, Arroyo Verruga, 1900 m (MUB 749); Santa María de Nieva, Sạ de la Estancia, $1129 \mathrm{~m}$ (MUB 1508); Los Lobos, Collado de la Casa Nueva, $370 \mathrm{~m}$ (MUB 751). JAEN: Sa de Segura, Molino del Prado de la Porra, $1200 \mathrm{~m}$ (MUB 7644); Sa de Segura, Collado Asperones, $1200 \mathrm{~m}$ (MUB 7649); Sa de Segura, Campamento Río Madera, $1300 \mathrm{~m}$ (MUB 7550, 7633, 7635, 7639). MURCIA: Bullas, Aceniche, $800 \mathrm{~m}$ (MUB 6009); Jumilla, Sa de1 Carche, Barranco del Revolcador, $900 \mathrm{~m}$ (MUB 6008); Cabo de Palos, $180 \mathrm{~m}$ (MUB 752 ).

Mayoritariamente corticicola, puede encontrarse en todo tipo de sustratos (rocas, restos óseos, epifilo, resinas...) ; nitrófilo; fotófilo.

8. Physcia aipolia (Humb.) Fürnrohr

ALBACETE: Riópar, Umbría Fuente Raigadas, $1400 \mathrm{~m}$ (MUB 7440); Riópar, Casa de la Noguera, 1000 m (MUB 7438, 7439); Riópar, Chorros del Mundo, $1000 \mathrm{~m}$ (MUB 7451, 7613); Riópar, Umbría Fuente Raigadas, $1000 \mathrm{~m}$ (MUB 7615). GRANADA: Sa Nevada, Bosque de Fuente Agrillos, $1400 \mathrm{~m}$ (MUB 1438). JAEN: Sa de Segura, Campamento Río Madera, $1300 \mathrm{~m}$ (MUB 7557); Sa de Segura, Collado Asperones, $1200 \mathrm{~m}$ (MUB 7646, 7647, 7651); Sa de Segura, Campamento Río Madera, $1300 \mathrm{~m}$ (MUB 7574, 7577). MURCIA: Jumilla, Sạ del Carche, Barranco del Revolcador, $900 \mathrm{~m}$ (MUB $6006,6007)$.

Corticicola, sobre todo tipo de forófitos, aunque prefiere los planifolios. Ocasionalmente se comporta como moderadamente acidófilo, hasta subeutrófilo, más o menos nitrófilo, fotófilo y toxitolerante. Sobre Quercus rotundifolia y Fraxinus angustifolia.

En cuanto a las características morfológicas, se pueden señalar las siguientes: 
1. Coloración variable de la cara inferior del talo, que va desde pardo a pardo oscuro, casi negro.

2. Presencia de proliferaciones foliáceas, que recuerdan a isidios en el margen de los lóbulos de algunos ejemplares examinados.

9. Physcia biziana (Massal.) Zahlbr.

AlBACETE: Riópar, Casa de la Noguera, $1000 \mathrm{~m}$ (MUB 7453). JAEN: $\mathrm{S}$ a de Segura, Embalse de Anchuricas, $1000 \mathrm{~m}$ (MUB 7595). MURCIA: Sa de Carrascoy, Rambla de Roy, 900 m (MUB 6010, 6011, 6014); Bullas, Aceniche, $800 \mathrm{~m}$ (MUB 6012); Jumilla, Sa del Carche, Solana de la Omblanquilla, $1000 \mathrm{~m}$ (MUB 6013).

Corticícola, ocasionalmente saxícola. Más o menos acidófilo, nitrotolerante. Sobre Quercus rotundifolia y Pinus halepensis.

Este ejemplar presenta unas ascósporas de tamaño algo mayor al dado por Clauzade (1970) para las de Ph. biziana. Próxima a Ph. stellaris, se separa de ésta por los lóbulos planos o cóncavos y el talo muy pruịoso.

10. Physcia caesia (Hoffm.) Fürnrohr

ALMERIA: Tabernas, Sa de Alhamilla, Colativi, $1300 \mathrm{~m}$ (MUB 1592); Uleila del Campo, Monteagudo, $1300 \mathrm{~m}$ (MUB 1588); Bacarés, Sa de los Filabres, Barranco Barrancón, 1700 m (MUB 1591 ); Gérgal, Sa de los Filabres, Arroyo Verruga, 1900 m (MUB $1590)$; Gérgal, Sa de los Filabres, Piedra del Sombrero, $2000 \mathrm{~m}$ (MUB 770, 1589); Alcaraz, Reolid, 1000-1100 m (MUB 1593).

Saxicola, ocasionalmente corticicola o lignicola. Crece tanto en sustratos ácidos como en básicos. Fotófila, xerófila, nitrófilo. Todo el material aqui citado es estéril.

11. Physcia clementei ( $\mathrm{Sm}$. ) Maas Geest.

JAEN: Sa de Segura, Collado Asperones, $1200 \mathrm{~m}$ (MUB 7540).

Corticicola. Sobre Ulmus sp

La distribución y dimensiones de los tejidos varian considerablemente desde la zona central a los extremos de los lóbulos. Asi, se puede encontrar desde una capa algal constituida por paquetes de células ordenadas perfectamente en el extremo, hasta una capa completamente irregular en la zona central de los lóbulos.

E1 ejemplar presenta caracteristicas que recuerdan bastante a Ph. aipolia. La presencia en ambas especies de abundantes puntos blancos en la cara superior del talo, asi como similares propiedades quimicas (médula $\mathrm{K}+$ ), ha llevado a que ambas especies sean confundidas. El material estudiado presenta diferencias suficientemente significativas para separar ambos táxones. Así, en Ph. clementei es muy caracteristica la presencia de pseudoisidios, muy abundantes en el centro del talo, los cuales no aparecen en Ph. aipolia. Por otra parte, Ph. clementei presenta la cara superior levemente cubierta de pruina blanca, localizada principalmente en el ápice de los lóbulos, mientras que Ph. aipolia carece de pruina. Los lóbulos de Ph. clementei son más anchos que los de Ph. aipolia.

12. Physcia dubia (Hoffm.) Lett.

AlBACETE: Alcaraz, Cerro de la Cueva, $1000 \mathrm{~m}$ (MUB 1597). ALMERIA: Tabernas, Sa Alhamilla, $1365 \mathrm{~m}$ (MUB 1598); Gérgal, Sa de los Filabres, Arroyo Verruga, $1900 \mathrm{~m}$ (MUB 1595); Bacarés, S de los Filabres, Collado del Conde, $1900 \mathrm{~m}$ (MUB 1594); Uleila 


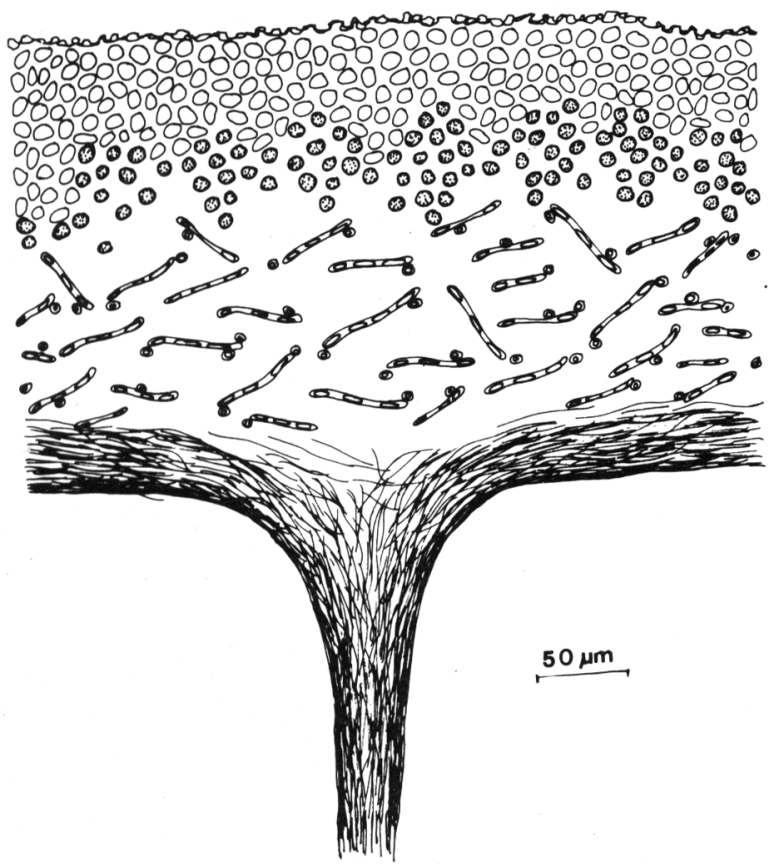

A

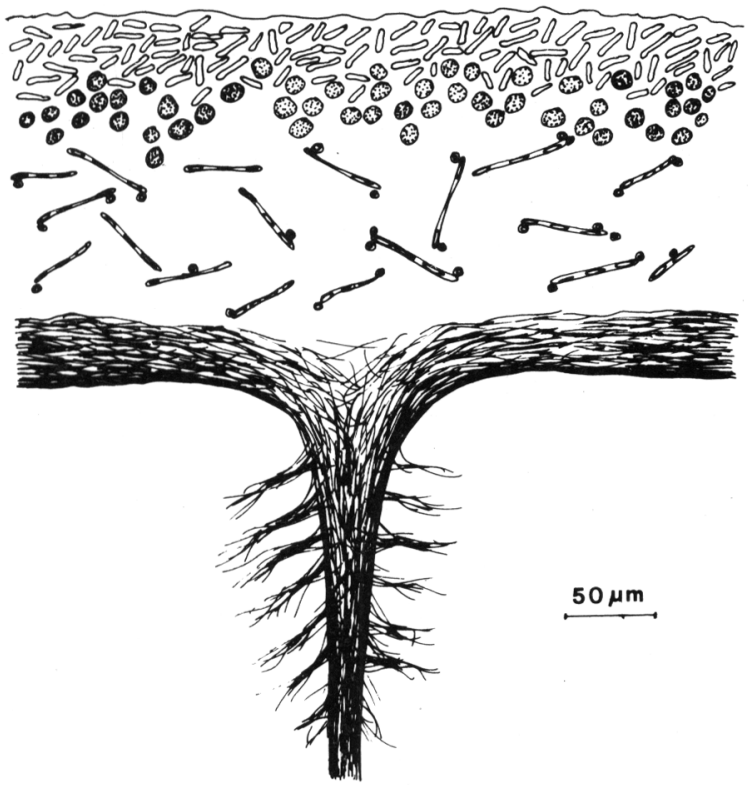

Figura 1: A. Physconia grisea. Sección longitudinal de un lóbulo. Córtex superior paraplectenquimático; córtex inferior prosoplectenquimático. Rizina simple. B: Physconia venusta. Sección longitudinal de un lóbulo. Córtex superior escleroplectenquimático; córtex inferior prosoplectenquimático. Rizina escuarrosa. 

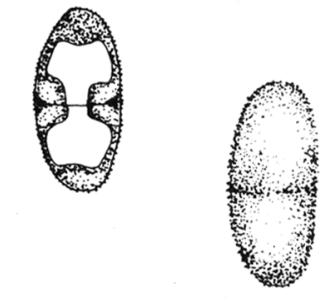

A

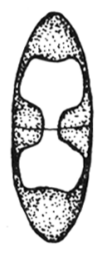

B

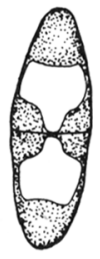

\section{$10 \mathrm{~mm}$}

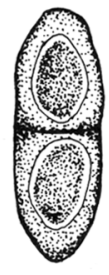

C
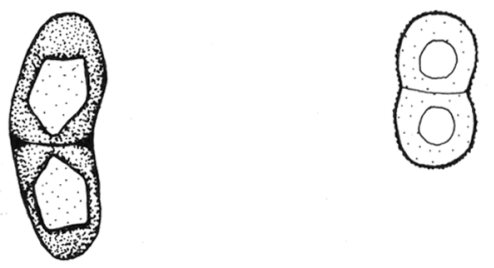

D

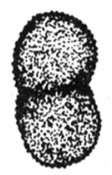

$15 \mu \mathrm{m}$

$10 \mu \mathrm{m}$
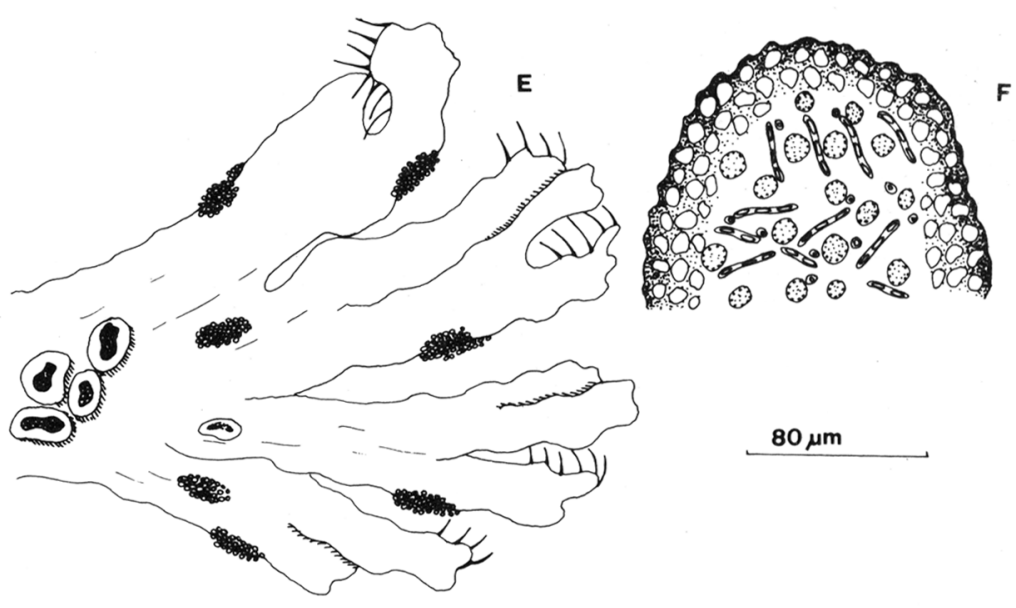

$1 \mathrm{~mm}$

Figura 2: A-B. Ascósporas tipo Physcia: A. Physcia magnussonii. B. Physcia stellaris. C. Ascósporas tipo Pachysporaria: Hyperphyscia adglutinata. D. Ascósporas tipo Physconia: Physconia pulverulacea. E. Phaeophyscia orbicularis: Soralios maculiformes y marginales.F. Physconia grisea. Sección de un isidio. 
del Campo, Monteagudo, $1300 \mathrm{~m}$ (MUB 1596). GRANADA: Sa Nevada, Veleta, $3200 \mathrm{~m}$ (MUB 1601); Sa N Nevada, Laguna de Aguas Verdes, $3000 \mathrm{~m}$ (MUB 1601); Sa Nevada, Laguna de Aguas Verdes, $3000 \mathrm{~m}$ (MUB 1600). MURCIA: Sạ de Carrascoy, W de 1a Cresta del Gallo, $300 \mathrm{~m}$ (MUB 1599).

Crece sobre todo tipo de sustratos. Sin embargo, se comporta principalmente como saxicola. Taxon muy nitrófilo, que en el SE de España coloniza las superficies rocosas, más o menos verticales, con fisuras por las que resbala el agua después de la lluvia. Heliófilo y fotófilo.

\section{Physcia magnussonii Frey}

ALMERIA: Tabernas, Sạ Alhamilla, Las Palomeras, $1200 \mathrm{~m}$ (MUB 747 ); Gérgal, Sa de los Filabres, Piedra del Sombrero, $2000 \mathrm{~m}$ (MUB 744); Bacarés, Sạ de los Filabres, Merendera, $1984 \mathrm{~m}$ (MUB 745); Gérgal, Sạ de los Filabres, Barranco del Pino, $1850 \mathrm{~m}$ (MUB 748); Gérgal, Sa de los Filabres, Barranco Barrancón, 1700 m (MUB 743). GRANADA: Sạ Nevada, Peñones de San Francisco, 2500 m (MUB 746).

Nitrófilo. Sobre paredes verticales o superverticales, en fisuras de roca y lugares protegidos.

14. Physcia semipinnata (Gmelin) Moberg

ALBACETE: Riópar, Umbria Fuente Raigadas, 1400 m (MUB 7446, 7448, 7449); Riópar, Chorros del Mundo, $1000 \mathrm{~m}$ (MUB 7437, 7443, 7444, 7445, 7450, 7617); Riópar, Arroyo de 1a Puerta, $1200 \mathrm{~m}$ (MUB 7447); Riópar, Casa de 1a Noguera, 1000 m (MUB 7439); Yeste, Casa del Barbero, $700 \mathrm{~m}$ (MUB 7610). ALMERIA: Tabernas, Sa Alhamilla, Pico Alhamilla, $1400 \mathrm{~m}$ (MUB 764). JAEN: Sa de Segura, Campamento Río Madera, 1300 m (MUB 7549, 7584, 7637, 7643 ); Sa de Segura, Embalse Anchuricas, $1000 \mathrm{~m}$ (MUB 7549). MURCIA: Sa de Carrascoy, $900 \mathrm{~m}$ (MUB 6001); Cartagena, Base del Roldán, 300 m (MUB 6004); Bullas, Aceniche, 800 m (MUB 6003); Bullas, Subida a la Sa de Burete, $600 \mathrm{~m}$ (MUB 6005); Jumilla, Sa del Carche, Solana de la Omblanquilla, $1000 \mathrm{~m}$ (MUB 6002 ).

Corticícola, más raramente lignicola o saxicola, nitrófilo, más o menos acidófilo, toxitolerante. Sobre Quercus rotundifolia, $Q$. coccifera, $Q$. pyrenaica, Ulmus glabra, Fraxinus angustifolia, Corylus avellana y Cistus clusii.

15. Physcia stellaris (L.) Nyl.

GRANADA: Sa Nevada, Bosque de Fuente Agrillos, 1400 m (MUB 1436). JAEN: Sa de Segura, Collado de los Asperones, $1200 \mathrm{~m}$ (MUB 7539); Sa de Segura, Embalse de Anchuricas, $1000 \mathrm{~m}$ (MUB $7593)$ :

Corticícola, generalmente sobre planifolios, menos frecuentemente sobre otros forófitos, raramente lignicola o saxícola. Sobre Quercus pyrenaica, Populus nigra y Ulmus sp.

16. Physcia tene11a (Scop.) DC.

ALBACETE: Alcaraz, Reolid, 1000-1100 m (MUB 762) Riópar, Cañada de los Mojones, $1400 \mathrm{~m}$ (MUB 7452); Yeste, Casa del Barbero, 700 m (MUB 7598). AlmERIA: Tabernas, Sa Alhamilla, Las Palomeras, $1200 \mathrm{~m}$ (MUB 750); Gérgal, Sa de los Filabres, Arroyo Verruga, $1900 \mathrm{~m}$ (MUB 763). JAEN: Sa de Segura, Campamento Río Madera, $1300 \mathrm{~m}$ (MUB 7619); Sa de Segura, Collado Asperones, $1200 \mathrm{~m}$ (MUB $7535,7648,7652)$.

Corticicola, raramente saxícola o lignícola; nitrófila. Sobre Quercus rotundifolia y olea europaea.

E1 carácter fundamental que presenta Ph. tene11a, y que 
permite separarla del resto de las especies de Physcia con cilios, es la presencia de soralios labriformes. En algunos casos, ejemplares de Ph. adscendens pueden ser determinados erróneamente como Ph. tene11a. Esto es debido a la rotura de los soralios en capuchón de la primera, tomando aspecto labriforme. También cuando presenta lóbulos anchos y cilios espaciados, Ph. tenella puede ser confundida con Ph. dubia; sin embargo, esta última tiene una superficie brillante y carente de verdaderos cilios.

17. Physconia enteroxantha (Ny1.) Poelt

ALBACETE: Alcaraz, Cañada del Conejo, $832 \mathrm{~m}$ (MUB 1584); Riópar, Chorros del Río Mundo, Lago de las Truchas, $1000 \mathrm{~m}$ (MUB 7435); Riópar, Casa de 1a Noguera, $1000 \mathrm{~m}$ (MUB 7436); Yeste, Casa de1 Barbero, $700 \mathrm{~m}$ (MUB 7599, 7600). ALMERIA: Sa Alhamilla, Colativi, $1365 \mathrm{~m}$ (MUB 1581); Gérgal, Sạ de los Filabres, Piedra del Sombrero, $2000 \mathrm{~m}$ (MUB 1376, 1586); Gérgal, Sa de los Filabres, Arroyo Verruga, $1900 \mathrm{~m}$ (MUB 1583); Bacarés, Sa de los Filabres, Merendera, $1984 \mathrm{~m}$ (MUB 1585). MURCIA: Sa de Carrascoy, Rambla de Roy, 900 m (MUB 6022).

Corticicola y saxícola, más o menos acidófilo; fotófilo; nitrófilo. Sobre Quercus rotundifolia y Populus nigra.

18a. Physconia grisea (Lam.) Poelt subsp. grisea

ALBACETE: Sa del Relumbrar, Cerro de la Cueva, $1000 \mathrm{~m}$ (MUB 773). Almeria: Uleila del Campo, Monteagudo, $1300 \mathrm{~m}$ (MUB 1577). Se comporta mayormente como corticícola, sobre planifolios, en la base de los troncos. Nitrófilo, más o menos fotófilo, toxitolerante. Se diferencia del resto de las especies del género en el tipo de rizinas, que son simples, y en el color de la cara inferior, algo más claro. Recolectado por nosotros en fisuras de rocas ácidas.

18b. Physconia grisea subsp. 1ilacina (Arnold) Poelt

ALMERIA: Gérga1, Sạ de los Filabres, Arroyo Verruga, $1900 \mathrm{~m}$ (MUB 7432 ).

Saxícola, tanto sobre rocas ácidas como básicas, menos frecuentemente lignicola o corticicola; nitrófilo, fotófilo y heliófilo.

Presenta un talo orbicular, de mayor tamaño (hasta $3,5 \mathrm{~cm}$ de anchura) que la especie tipo. Lóbulos más estrechos $(0,9-1,9$ $\mathrm{mm}$ ). Abundantemente cubierta de pruina blanca, de cierta tonalidad lilacina.

19. Physconia perisidiosa (Erichs.) Moberg

ALBACETE: Alcaraz, Cañada del Conejo, $832 \mathrm{~m}$ (MUB 171); Alcaraz, Reolid, 1000-1100 m (MUB 1579); Riópar, Casa de la Noguera, $1000 \mathrm{~m}$ (MUB 7433, 7434). ALMERIA: Tabernas, Sa alhamilla, Pico Alhamilla, $1365 \mathrm{~m}$ (MUB 1570); Gérgal, Sa de los Filabres, Piedra del Sombrero, $2000 \mathrm{~m}$ (MUB 1407); Bacarés, Merendera, 1984 m (MUB 1574); Gérgal, Sạ de los Filabres, Barranco del Pino, $1850 \mathrm{~m}$ (MUB 1573). GRANADA: Sạ Nevada, Bosque Fuente Agrillos, $1400 \mathrm{~m}$ (MUB 1433); Guadix, $12000 \mathrm{~m}$ (MUB 1572). JAEN: Sa de Segura, Collado Asperones, $1200 \mathrm{~m}$ (MUB 7542, 7650); Sa de Segura, Campamento Río Madera, 1300 m (MUB 7636, 7640). MURCIA: Sa de Carrascoy, Rambla de Roy, 900 m (MUB 6023); Bullas, Cerro del Lomillo, $1000 \mathrm{~m}$ (MUB 772); Jumilla, Sa del Carche, Umbría de1 Mosquito, (MUB 6024).

Taxon predominantemente corticicola, sobre planifolios. Frecuentemente se ha observado también en las fisuras de roca 

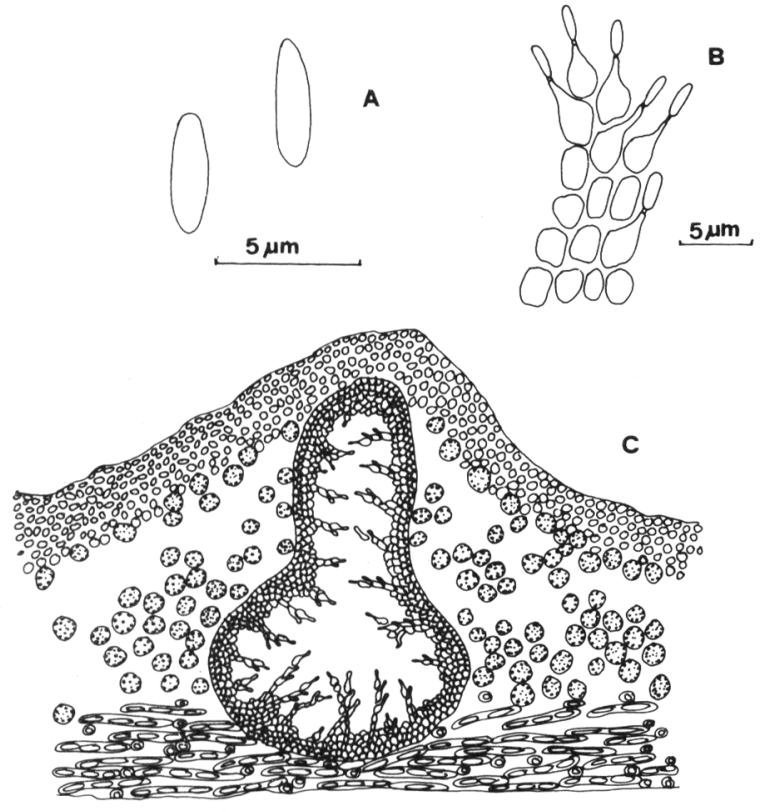

$25 \mu \mathrm{m}$

$100 \mu m$
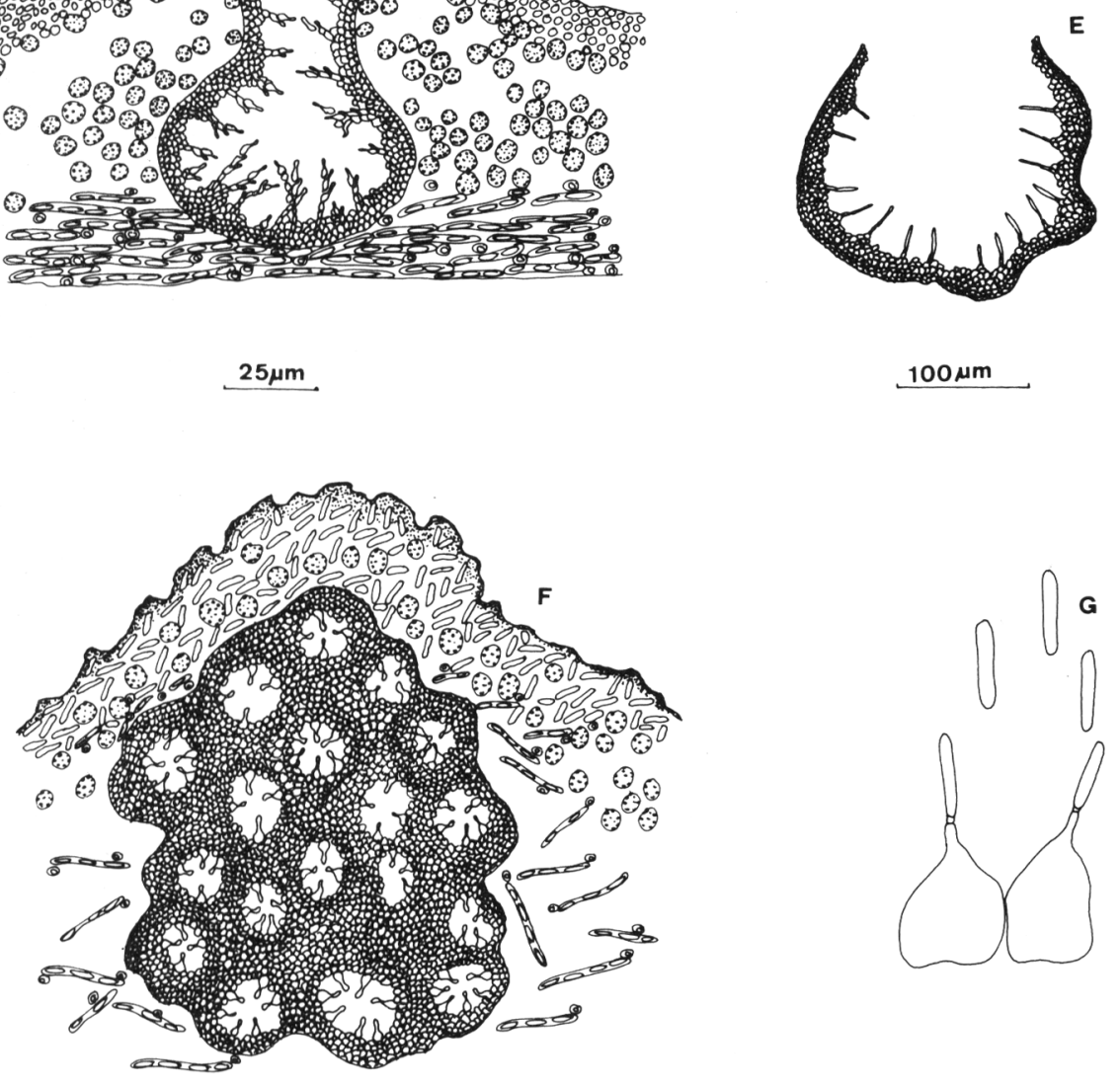

$5 \mu \mathrm{m}$

$100 \mu \mathrm{m}$

Figura 3: A-B. Phaeophyscia ciliata. A. Picnoconidios elipsoidales. B. Conidióforos. C. Picnidio tipo Umbilicaria. D-E. Hyperphyscia adglutinata. D. Conidióforos y conidios filiformes. E. Picnidio tipo Roccella. F-G. Physconia pulverulacea. F. Picnidio tipo Xanthoria. G. Conidióforos y conidios cilíndricos. 
con abundancia de tierra. Más o menos acidófilo, fotófilo y nitrófilo. Sobre Quercus rotundifolia, $\boldsymbol{Q}$. faginea, Ulmus sp y Pinus nigra.

20. Physconia pulverulacea Moberg

ALBACETE: Riópar, Chorros del Mundo, $1000 \mathrm{~m}$ (MUB 7421, 7422, 7424, 7426, 7427, 7428, 7429); Riópar, Lago de los Truchas, $1000 \mathrm{~m}$ (MUB 7425, 7430); Riópar, Umbría Fuente Raigadas, $1400 \mathrm{~m}$ (MUB 7431). GRANADA: Sa Nevada, Bosque Fuente Agrillos, $1400 \mathrm{~m}$ (MUB 1434).

Corticícola, en ocasiones se comporta como saxícola, sobre todo en fisuras donde se acumulan tierra y humus. Más o menos acidófilo, fotófilo, nitrófilo, toxitolerante. Sobre Quercus rotundifolia, Q. pyrenaica, Q. faginea, Ulmus glabra y Fraxinus angustifolia.

21. Physconia venusta (Ach.) Poelt

ALBACETE: Riópar, Umbria Fuente Raigadas, 1400 m (MUB 7408, 7412, 7416, 7419, 7420); Riópar, Carretera de los Chorros, 1000 m (MUB 7407); Riópar, Chorros del Mundo, 1000 m (MUB 7409, 7417, 7614); Riópar, Lago de las Truchas, $100 \mathrm{~m}$ (MUB 7414). ALMERIA: Tabernas, Sạ Alhamilla, Pico alhamilla, $1365 \mathrm{~m}$ (MUB 1578). JAEN: Sa de Segura, Campamento Río Madera, $1300 \mathrm{~m}$ (MUB 7543, 7569, 7634). MURCIA: Sạ de Carrascoy, Rambla de Roy, 900 m (MUB 6021).

Corticícola, sobre planifolios en general; ocasionalmente en fisuras de rocas, donde se acumula tierra y humus (terrícola, humícola). En general, nitrófilo y fotófilo. Sobre Q. rotundifolia, Q. faginea, Q. pyrenaica, Ilex aquifolium, Ulmus glabra, Corylus avellana, Crataegus monogyna, Populus nigra y Pinus nigra.

\section{BIBLIOGRAFIA}

CHOISY, M. -1950- Catalogue des lichens de la region Lyonnaise. Bull. Soc. Linn. Lyon 19:9-24.

CRESPO, A \& BUENO, A.G. -1982- Flora y vegetación liquénicas de la Casa de Campo de Madrid (España). Lazaroa, 4:327-356.

FREY, E. -1963- Beiträge zu einer Lichenenflora des Schweiz. Bot. Ges. 73:389-503.

HAFELLNER, J. et al. -1979- Die Gattungen der Fletchenfamilie Physciaceae. Herzogia 5:39-79.

KASHIWADANI, H. -1975- The genera Physcia, Physconia and Dirinaria of Japan. Ginkgoana 3.

LYNGE, B. -1916- A monograph of the Norwegian Physciaceae. Vid. Selsk. Skriften I. Mat. Naturv. kl. n. 8. Christiania.

LYNGE, B. -1935- Physciaceae. Rabenhorst Krypt. 2 ed., IX, 6(1):41-188.

MAAS GESTERANUS, R.A. -1952- Revision of the lichens of the Netherlands. II. Physciaceae. Blumea $7(1): 206-287$.

MICHAUX, A. -1803- Flora boreali-americana. II. Parisiis et Argentorati. MOBERG, R. -1974- Studies on Physcia I. Sv. Bot. Tidskr. 68:285-288. MOBERG, R. -1975- Studies on Physcia II. Sv. Bot. Tidskr. 69:67-71. MOBERG, R. -1977- The lichen genus Physcia and allied genera in Fennoscandia. Symb. Bot. Uppsal. XXII:1-108.

MULLER, J. -1884- Conspectus systematicus lichenus Novae Zelandiae quem elaborativ. Bull. Herb. Boissier 2, App. 1:1-114.

NADVORNIK, J. -1947- Physciaceae Tchécoslovaques. Stud Bot. Cechoslovaca $8: 69-124$. 
OZENDA, P. \& CLAUZADE, G. -1970- Les 1ichens. Masson. Paris.

POELT, J. -1965- Uber einige Artengruppen der Flechtengattungen Caloplaca und Fulgensia. Mitt. Bot. Staatssammlung München 5:571-607.

POELT, J. -1969- Bestimmungschlüssel Europäischer Flechten. J. Cramer. Lehre.

POELT, J. -1974- Die Gattungen Physcia, Physciopsis und Physconia. Khumbu Himal. 6:57-100.

THOMSON, J.W. -1963- The lichen genus Physcia in North America. Nova Hedwigia $7: 1-172$. 